\title{
ON THE STRUCTURE OF THE SET OF PERIODS FOR PERIODIC SOLUTIONS OF SOME LINEAR INTEGRO-DIFFERENTIAL EQUATIONS ON THE MULTIDIMENSIONAL SPHERE
}

\author{
DANG KHANH HOI
}

\begin{abstract}
The problem of periodic solutions for the family of linear differential equations

$$
(L-\lambda) u \equiv\left(\frac{1}{i} \frac{\partial}{\partial t}-a \Delta-\lambda\right) u(x, t)=\nu G(u-f)
$$

is considered on the multidimensional sphere $x \in S^{n}$ under the periodicity condition $\left.u\right|_{t=0}=\left.u\right|_{t=b}$. Here $a$ and $\lambda$ are given reals, $\nu$ is a fixed complex number, $G u(x, t)$ is a linear integral operator, and $\Delta$ is the Laplace operator on $S^{n}$. It is shown that the set of parameters $(\nu, b)$ for which the above problem admits a unique solution is a measurable set of full measure in $\mathbb{C} \times \mathbb{R}^{+}$.
\end{abstract}

$\S 1$

In [4, 5] it was discovered that, for some partial differential equations, the set of periods for which a periodic solution is unique may have an unexpectedly complicated structure. In this paper, we study this issue for a class of linear equations on the multidimensional sphere.

We consider the problem of periodic solutions for the nonlocal Schrödinger type equation

$$
\left(\frac{1}{i} \frac{\partial}{\partial t}-a \Delta-\lambda\right) u(x, t)=\nu G(u-f)
$$

with the $t$-periodicity condition

$$
\left.u\right|_{t=0}=\left.u\right|_{t=b} .
$$

Here $u(x, t)$ is a complex function on $S^{n} \times[0, b]$, where $S^{n}$ is the multidimensional sphere, $n \geq 2 ; a \neq 0, \lambda$, and $\nu$ are given complex numbers; $f(x, t)$ is a given function.

The change of variables $t=b \tau$ reduces our problem to a problem with a fixed period, but with a new equation in which the coefficient of the $\tau$-derivative is equal to $\frac{1}{b}$,

$$
\left(\frac{1}{i} \frac{\partial}{b \partial \tau}-a \Delta-\lambda\right) u(x, b \tau)=\nu G(u(x, b \tau)-f(x, b \tau)) .
$$

$\S 2$

Thus, problem (10), (2) turns into a problem on periodic solutions of the equation

$$
(L-\lambda) u \equiv\left(\frac{1}{i} \frac{\partial}{b \partial t}-a \Delta-\lambda\right) u(x, t)=\nu G(u-f)
$$

with the fixed periodicity condition

$$
\left.u\right|_{t=0}=\left.u\right|_{t=1} .
$$

2000 Mathematics Subject Classification. Primary 35K20.

Key words and phrases. Schrödinger-type equation, periodicity condition. 
Here $G u(x, t)=\int_{S_{n}} g(x, y) u(y, t) d y$ (dy is the Lebesgue-Hausdorff measure on the sphere $\left.S^{n}\right)$ is an integral operator on the space $L_{2}\left(S^{n} \times[0,1]\right)$ with smooth kernel $g(x, y)$ defined on $S^{n} \times S^{n}$. The differential operation $\frac{1}{i} \frac{\partial}{b \partial t}-a \Delta$ is assumed to be defined on the functions $u(x, t) \in C^{\infty}\left(S^{n} \times[0,1]\right)$ such that $\left.u\right|_{t=0}=\left.u\right|_{t=1}$. Let $L$ denote the closure of this operation $\frac{1}{i} \frac{\partial}{b \partial t}-a \Delta$ in $\mathcal{H}=L_{2}\left(S^{n} \times[0,1]\right)$. So, an element $u \in \mathcal{H}$ belongs to the domain $\mathcal{D}(L)$ of $L=\frac{1}{i} \frac{\partial}{b \partial t}-a \Delta$ if and only if there is a sequence $\left\{u_{j}\right\} \subset C^{\infty}\left(S^{n} \times[0,1]\right)$, $\left.u_{j}\right|_{t=0}=\left.u_{j}\right|_{t=1}$, such that $\lim u_{j}=u, \lim L u_{j}=L u$ in $\mathcal{H}$.

It is well known that the eigenvalues of the Laplace operator $\Delta$ on the sphere $S^{n}$ are of the form $-k(k+n-1), k \in \mathbb{Z}, k \geq 0$, and that $\Delta$ admits the corresponding orthonormal basis of eigenfunctions $w_{k}(x) \in C^{\infty}\left(S^{n}\right)$ (see, e.g., [3]).

Lemma 1. The functions $e_{k m}(x, t)=e^{i 2 \pi m t} w_{k}(x), k, m \in \mathbb{Z}, k \geq 0$, are eigenfunctions of the operator $L$ in the space $\mathcal{H}=L_{2}\left(S^{n} \times[0,1]\right)$ that correspond to the eigenvalues

$$
\lambda_{k m}=\frac{2 m \pi}{b}+a k(k+n-1)=\frac{2 m \pi}{b}+\lambda_{k} .
$$

These functions form an orthonormal basis in $\mathcal{H}$. The domain of $L$ is given by the formula

$$
\mathcal{D}(L)=\left\{u=\left.\sum u_{k m} e_{k m}\left|\sum\right| \lambda_{k m} u_{k m}\right|^{2}<\infty, \sum\left|u_{k m}\right|^{2}<\infty\right\} .
$$

The spectrum $\sigma(L)$ is the closure of the set $\left\{\lambda_{k m}\right\}$.

Lemma 2.

$$
\|G\|^{2} \leq M_{0}^{2}=\int_{S^{n}} \int_{S^{n}}|g(x, y)|^{2} d x d y .
$$

Proof. We have

$$
\begin{aligned}
|G u(x, t)|^{2} & =\left|\int_{S^{n}} g(x, y) u(y, t) d y\right|^{2} \leq \int_{S^{n}}|g(x, y)|^{2} d y \int_{S^{n}}|u(y, t)|^{2} d y, \\
\|G u(x, t)\|^{2} & =\int_{0}^{1} \int_{S^{n}}|G u(x, t)|^{2} d x d t \\
& \leq \int_{0}^{1} \int_{S^{n}}\left(\int_{S^{n}}|g(x, y)|^{2} d y \int_{S^{n}}|u(y, t)|^{2} d y\right) d x d t \\
\|G u(x, t)\|^{2} & \leq \int_{S^{n}} \int_{S^{n}}|g(x, y)|^{2} d x d y \int_{S^{n}} \int_{0}^{1}|u(y, t)|^{2} d y d t=M_{0}^{2}\|u\|^{2}, \\
\|G\| & \leq M_{0}
\end{aligned}
$$

The lemma is proved.

We note that the Laplace operator is formally selfadjoint relative to the scalar product $(u, v)=\int_{S^{n}} u(x) \overline{v(x)} d x$ on the space $C^{\infty}\left(S^{n}\right)$. The product $\Delta_{x} \circ G=\Delta_{x} G$ coincides with the integral operator with the kernel $\Delta_{x} g(x, y)$. We put $M=\max \left\{\left\|\Delta_{x} G\right\|,\|G\|\right\}$.

Lemma 3. Let $v=G u=\sum v_{k m} e_{k m} ;$ then

$$
\left|v_{k m}\right|^{2} \leq \frac{4 M^{2}}{(k(k+n-1)+1)^{2}}\|u\|^{2} .
$$

Also, for $k \neq 0$ we have

$$
\left|v_{k m}\right|^{2} \leq \frac{4\left|\alpha_{k m}\right|^{2}}{(k(k+n-1)+1)^{2}},
$$

where $\alpha_{k m}=\left\langle\Delta_{x} G u, e_{k m}\right\rangle$, and $\sum\left|\alpha_{k m}\right|^{2} \leq M^{2}\|u\|^{2}$. 
Proof. The Parseval identity $\sum\left|v_{k m}\right|^{2}=\|G u\|^{2}$ yields

$$
\left|v_{0 m}\right|^{2} \leq\|G\|^{2}\|u\|^{2} \leq 4 M^{2}\|u\|^{2} .
$$

Since the Laplace operator is selfadjoint, for $k \neq 0$ we have

$$
\begin{aligned}
& \alpha_{k m}=\left\langle\Delta_{x} G u, e_{k m}\right\rangle=\left\langle G u, \Delta_{x} e_{k m}(x, t)\right\rangle=\left\langle G u,-k(k+n-1) e_{k m}(x, t)\right\rangle, \\
& \alpha_{k m}=-k(k+n-1)\left\langle G u, e_{k m}(x, t)\right\rangle=-k(k+n-1) v_{k m} .
\end{aligned}
$$

It follows that

$$
\left|v_{k m}\right|^{2}=\frac{\left|\alpha_{k m}\right|^{2}}{(k(k+n-1))^{2}} \leq \frac{4\left|\alpha_{k m}\right|^{2}}{(k(k+n-1)+1)^{2}} .
$$

By the Parseval identity, we have $\sum\left|\alpha_{k m}\right|^{2}=\left\|\Delta_{x} G u\right\|^{2} \leq M^{2}\|u\|^{2}$, whence

$$
\left|v_{k m}\right|^{2} \leq \frac{4 M^{2}\|u\|^{2}}{(k(k+n-1)+1)^{2}}
$$

The lemma is proved.

We assume that $a$ and $\lambda$ are real numbers. Then, by Lemma 1, the spectrum $\sigma(L)$ lies on the real axis. Most typical and interesting is the case where the number $a b /(2 \pi)$ is irrational. The H. Weyl theorem (see, e.g., 1]) says that, in this case, the set of the numbers $\lambda_{k m}$ is everywhere dense on $\mathbb{R}$ and $\sigma(L)=\mathbb{R}$. Now, suppose that $\lambda \neq \lambda_{k m}$ for all $k, m \in \mathbb{Z}, k \geq 0$. Then the inverse operator $(L-\lambda)^{-1}$ is well defined, but unbounded. The expression for this inverse operator involves small denominators:

$$
(L-\lambda)^{-1} v(x, t)=\sum \frac{v_{k m}}{\lambda_{k m}-\lambda} e_{k m},
$$

where the $v_{k m}$ are the Fourier coefficients of the series

$$
v(x, t)=\sum_{k, m \in \mathbb{Z}, k \geq 0} v_{k m} e_{k m}
$$

For positive numbers $\sigma$ and $C$, let $A_{\sigma}(C)$ denote the set of all positive $b$ such that

$$
\left|\lambda_{k m}-\lambda\right| \geq \frac{C}{(k+1)^{1+\sigma}}
$$

for all $m, k \in \mathbb{Z}, k \geq 0$.

This definition shows that the sets $A_{\sigma}(C)$ extend as $C$ reduces and as $\sigma$ grows. Therefore, in what follows, to prove that such a set or its part is nonempty, we require that $C$ be sufficiently small and $\sigma$ sufficiently large. Let $A_{\sigma}$ denote the union of the sets $A_{\sigma}(C)$ over all $C>0$.

If inequality (8) is fulfilled for some $b$ and all $m, k$, then it is fulfilled for $m=0$; this provides a condition necessary for the nonemptiness of $A_{\sigma}(C)$ :

$$
C \leq(k+1)^{1+\sigma}|a k(k+n-1)-\lambda|, \quad \forall k \geq 0 .
$$

We put $d=\min _{k \in \mathbb{Z}, k \geq 0}(k+1)^{1+\sigma}|a k(k+n-1)-\lambda|>0$.

Theorem 1. The sets $A_{\sigma}(C)$ and $A_{\sigma}$ are Borel. The set $A_{\sigma}$ has full measure, i.e., its complement to the half-line $\mathbb{R}^{+}$is of zero measure.

Proof. Obviously, the sets $A_{\sigma}(C)$ are closed in $\mathbb{R}^{+}$. The set $A_{\sigma}=\bigcup_{r=1}^{\infty} A_{\sigma}(1 / r)$ is Borel, being a countable union of closed sets. We show that $A_{\sigma}$ has full measure in $\mathbb{R}^{+}$. Suppose $b, l>0$ and $C \leq \frac{d}{2}$; we consider the complement $(0, l) \backslash A_{\sigma}(C)$. This set consists of all positive numbers $b$ for which there exist $m$ and $k$ such that

$$
\left|\lambda_{k m}-\lambda\right|<\frac{C}{(k+1)^{1+\sigma}}
$$


Solving this inequality for $b$, we see that, for $m, k$ fixed, the numbers $b$ form an interval $I_{k, m}=\left(m \alpha_{k}, m \beta_{k}\right)$, where $m=1,2,3, \ldots$,

$$
\begin{aligned}
\alpha_{k} & =\frac{2 \pi}{|a k(k+n-1)-\lambda|+\frac{C}{(k+1)^{1+\sigma}}}, \\
\beta_{k} & =\frac{2 \pi}{|a k(k+n-1)-\lambda|-\frac{C}{(k+1)^{1+\sigma}}} .
\end{aligned}
$$

The length of $I_{k, m}$ is $m \delta_{k}$, with

$$
\delta_{k}=\frac{4 \pi C(k+1)^{-1-\sigma}}{|a k(k+n-1)-\lambda|^{2}-C^{2}(k+1)^{-2-2 \sigma}} .
$$

Since $C \leq \frac{d}{2}$ by assumption, we have

$$
\delta_{k} \leq \frac{16 \pi C}{3(k+1)^{1+\sigma}|a k(k+n-1)-\lambda|^{2}} .
$$

For $k$ fixed and $m$ varying, there are only finitely many intervals $I_{k, m}$ that intersect the given segment $(0, l)$.

Such intervals arise for the values of $m=1,2, \ldots$ satisfying $m \alpha_{k}<l$, i.e.,

$$
0<m<\frac{l}{2 \pi}\left(|a k(k+n-1)-\lambda|+C(k+1)^{-1-\sigma}\right) .
$$

Since $C(k+1)^{-1-\sigma} \leq \frac{1}{2}|a k(k+n-1)-\lambda|$, we can write simpler restrictions on $m$ :

$$
0<m<\frac{l}{2 \pi} \frac{3}{2}|a k(k+n-1)-\lambda|<\frac{l}{\pi}|a k(k+n-1)-\lambda| .
$$

The measure of the intervals indicated (for $k$ fixed) is dominated by $\delta_{k} \widetilde{S}_{k}$, where $\widetilde{S}_{k}=$ $\widetilde{S}_{k}(l)$ is the sum of all integers $m$ satisfying (12). Summing the arithmetic progression, we obtain

$$
\widetilde{S}_{k} \leq \frac{l}{2 \pi^{2}}|a k(k+n-1)-\lambda|\{l|a k(k+n-1)-\lambda|+\pi\} .
$$

Passing to the union of the intervals in question over $k$ and $m$ and using (11), we see that

$$
\mu\left((0, l) \backslash A_{\sigma}(C)\right) \leq \sum_{k=0}^{\infty} \delta_{k} \widetilde{S}_{k} \leq C S(l)
$$

where

$$
S=S(l)=\sum_{k=0}^{\infty} \frac{8 l\{l|a k(k+n-1)-\lambda|+\pi\}}{3 \pi(k+1)^{1+\sigma}|a k(k+n-1)-\lambda|} .
$$

Observe that the quantity

$$
\frac{l|a k(k+n-1)-\lambda|+\pi}{\pi|a k(k+n-1)-\lambda|}
$$

is dominated by a constant $D$; therefore,

$$
S(l) \leq \frac{8}{3} l D \sum_{k=0}^{\infty} \frac{1}{(k+1)^{1+\sigma}}<\infty .
$$

We have

$$
\mu\left((0, l) \backslash A_{\sigma}\right) \leq \mu\left((0, l) \backslash A_{\sigma}(C)\right) \leq C S(l)
$$

for all $C>0$. It follows that $\mu\left((0, l) \backslash A_{\sigma}\right)=0$ for all $l>0$. Thus, $\mu\left((0, \infty) \backslash A_{\sigma}\right)=0$ and $A_{\sigma}$ has full measure. The theorem is proved. 
Theorem 2. Suppose $g(x, y)$ is a function defined on $S^{n} \times S^{n}$ and such that the function $\Delta_{x} g(x, y)$ is continuous on $S^{n} \times S^{n}$. Let $0<\sigma<1$, and let $b \in A_{\sigma}(C)$. Then the inverse operator $(L-\lambda)^{-1}$ is well defined, and the operator $(L-\lambda)^{-1} \circ G$ is compact.

Proof. Since $b \in A_{\sigma}(C)$, we have $\lambda_{k m} \neq \lambda$ for all $k, m \in \mathbb{Z}, k \geq 0$, so that $(L-\lambda)^{-1}$ is well defined and looks like the expression in (7). Observe that $\lim \frac{(k+1)^{2+2 \sigma}}{(k(k+n-1)+1)^{2}}=0$ as $k \rightarrow \infty$. Therefore, given $\varepsilon>0$, we can find an integer $k_{0}>0$ such that

$$
\frac{(k+1)^{2+2 \sigma}}{(k(k+n-1)+1)^{2}}<\frac{(\varepsilon C)^{2}}{(2 M)^{2}}
$$

for all $k>k_{0}$.

We write

$$
(L-\lambda)^{-1} v(x, t)=Q_{k_{0} 1} v+Q_{k_{0} 2} v, \quad v=G u,
$$

where

$$
Q_{k_{0} 1} v=\sum_{0 \leq k \leq k_{0}} \frac{v_{k m}}{\lambda_{k m}-\lambda} e_{k m}, \quad Q_{k_{0}} v=\sum_{k>k_{0}} \frac{v_{k m}}{\lambda_{k m}-\lambda} e_{k m} .
$$

For the operator $Q_{k_{0} 1}$ we have

$$
\left\|Q_{k_{0} 1} v\right\|^{2}=\sum_{0 \leq k \leq k_{0}} \frac{\left|v_{k m}\right|^{2}}{\left|\lambda_{k m}-\lambda\right|^{2}} .
$$

Observe that if $0 \leq k \leq k_{0}$, then

$$
\lim \frac{1}{\left|\frac{2 m \pi}{b}+a k(k+n-1)-\lambda\right|^{2}}=0
$$

as $|m| \rightarrow \infty$. Therefore, the quantity $\frac{1}{\left|\frac{2 m \pi}{b}+a k(k+n-1)-\lambda\right|^{2}}$ is dominated by a constant $C\left(k_{0}\right)$. Then

$$
\left\|Q_{k_{0} 1} v\right\|^{2} \leq \sum\left|v_{k m}\right|^{2} C\left(k_{0}\right) \leq C\left(k_{0}\right)\|v\|^{2},
$$

which means that $Q_{k_{0} 1}$ is a bounded operator.

Consider the operator $Q_{k_{0} 2} \circ G$. By 3 and Lemma (8), we have

$$
\begin{aligned}
\left\|Q_{k_{0}} v\right\|^{2} & =\left\|Q_{k_{0} 2} \circ G u\right\|^{2}=\sum_{k>k_{0}} \frac{\left|v_{k m}\right|^{2}}{\left|\lambda_{k m}-\lambda\right|^{2}} \\
& \leq \sum_{k>k_{0}} \frac{4 \alpha_{k m}^{2}}{(k(k+n-1)+1)^{2}}\left(\frac{1}{C}\right)^{2}(k+1)^{2+2 \sigma} \\
& \leq\left(\frac{1}{C}\right)^{2}\left(\frac{\varepsilon C}{2 M}\right)^{2} \sum_{k>k_{0}} 4\left|\alpha_{k m}\right|^{2} \leq \varepsilon^{2}\|u\|^{2} .
\end{aligned}
$$

Consequently, $\left\|Q_{k_{0} 2} \circ G\right\| \leq \varepsilon$.

Since $G$ is compact and $Q_{k_{0} 1}$ is bounded, $Q_{k_{0} 1} \circ G$ is compact. Next, we have

$$
\left\|(L-\lambda)^{-1} \circ G-Q_{k_{0} 1} \circ G\right\|=\left\|Q_{k_{0}} \circ G\right\|<\varepsilon .
$$

Thus, the operator $(L-\lambda)^{-1} \circ G$ is the limit of a sequence of compact operators. Therefore, it is compact itself. The theorem is proved.

We denote $K=K_{b}=(L-\lambda)^{-1} \circ G$.

Theorem 3. Suppose $b \in A_{\sigma}(C)$. Then problem (11), (2) admits a unique periodic solution with period $b$ for all $\nu \in \mathbb{C}$ except, possibily, an at most countable discrete set of values of $\nu$. 
Proof. Equation (11) reduces to

$$
\left((L-\lambda)^{-1} \circ G-\frac{1}{\nu}\right) u=(L-\lambda)^{-1} \circ G(f) .
$$

We write $(L-\lambda)^{-1} \circ G-\frac{1}{\nu}=K-\frac{1}{\nu}$.

Since $K$ is a compact operator, its spectrum $\sigma(K)$ is at most countable, and the limit point of $\sigma(K)$ (if any) can only be zero. Therefore, the set $S=\left\{\nu \neq 0 \mid \frac{1}{\nu} \in \sigma(K)\right\}$ is at most countable and discrete, and if $\nu \neq 0$ and $\nu \notin S$, then the operator $\left(K-\frac{1}{\nu}\right)$ is invertible, i.e., equation (1) is uniquely solvable. The theorem is proved.

We pass to the question of the solvability of problem (1), (2) for fixed $\nu$. We need to study the structure of the set $E \subset \mathbb{C} \times \mathbb{R}^{+}$that consists of all pairs $(\nu, b)$ with $\nu \neq 0$ and $\frac{1}{\nu} \notin \sigma\left(K_{b}\right)$, where $K_{b}=(L-\lambda)^{-1} \circ G$.

Theorem 4. E is a measurable set of full measure in $\mathbb{C} \times \mathbb{R}^{+}$.

For the proof, we need several auxiliary statements.

Lemma 4. For any $\varepsilon>0$, there exists an integer $k_{0}$ such that $\left\|K_{b}-\widetilde{K_{b}}\right\|<\varepsilon$ for all $b \in A_{\sigma}\left(\frac{1}{r}\right), 0<\sigma<1$, where $r=1,2, \ldots$,

$$
K_{b} u=\left(L_{b}-\lambda\right)^{-1} v=\sum \frac{v_{k m}}{\lambda_{k m}(b)-\lambda} e_{k m}, \quad \widetilde{K_{b}} u=\sum_{0 \leq k \leq k_{0}} \frac{v_{k m}}{\lambda_{k m}(b)-\lambda} e_{k m} .
$$

Proof. Observe that for any $\varepsilon>0$ there is an integer $k_{0}$ such that

$$
\frac{(k+1)^{2+2 \sigma}}{(k(k+n-1)+1)^{2}} \leq\left(\frac{\varepsilon}{2 r M}\right)^{2}
$$

for all $k \geq k_{0}, 0<\sigma<1$. We have

$$
\begin{aligned}
\left(K_{b}-\widetilde{K_{b}}\right) u & =K_{k_{0} b} u=\sum_{k>k_{0}} \frac{v_{k m}}{\lambda_{k m}(b)-\lambda} e_{k m}, \\
\left\|\left(K_{b}-\widetilde{K_{b}}\right) u\right\|^{2} & =\left\|K_{k_{0} b} u\right\|^{2}=\sum_{k>k_{0}}\left|\frac{v_{k m}}{\lambda_{k m}(b)-\lambda}\right|^{2} \\
& \leq \sum_{k>k_{0}} \frac{4 r^{2} \alpha_{k m}^{2}(k+1)^{2+2 \sigma}}{(k(k+n-1)+1)^{2}} \\
& \leq r^{2}\left(\frac{\varepsilon}{2 r M}\right)^{2} 4 \sum_{k>k_{0}}\left|\alpha_{k m}\right|^{2} \\
& \leq r^{2}\left(\frac{\varepsilon}{2 r M}\right)^{2} 4 M^{2}\|u\|^{2}=\varepsilon^{2}\|u\|^{2} .
\end{aligned}
$$

Thus, $\left\|K_{b}-\widetilde{K_{b}}\right\|=\left\|K_{k_{0} b}\right\|<\varepsilon$, as required.

Lemma 5. The operator-valued function $b \mapsto K_{b}$ is continuous for $b \in A_{\sigma}\left(\frac{1}{r}\right)$.

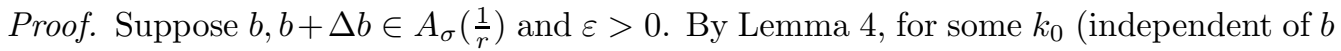
and $b+\Delta b$ ), we have $\left\|K_{b}-\widetilde{K_{b}}\right\|=\left\|K_{k_{0} b}\right\|<\varepsilon$ and $\left.\left\|K_{b+\Delta b}-\widetilde{K_{b+\Delta b}}\right\|=\| K_{k_{0}(b+\Delta b}\right) \|<\varepsilon$. Next,

$$
K_{b+\Delta b}-K_{b}=\left(\widetilde{K_{b+\Delta b}}+K_{k_{0}(b+\Delta b)}\right)-\left(\widetilde{K_{b}}+K_{k_{0} b}\right),
$$

whence we obtain

$$
\left\|K_{b+\Delta b}-K_{b}\right\| \leq\left\|\widetilde{K_{b+\Delta b}}-\widetilde{K_{b}}\right\|+\left\|K_{k_{0}(b+\Delta b)}\right\|+\left\|K_{k_{0} b}\right\| .
$$


Consider the operators $\widetilde{K_{b+\Delta b}}$ and $\widetilde{K_{b}}$. We have

$$
\begin{aligned}
\left(\widetilde{K_{b+\Delta b}}-\widetilde{K_{b}}\right) u & =\sum_{0 \leq k \leq k_{0}}\left(\frac{1}{\lambda_{k m}(b+\Delta b)-\lambda}-\frac{1}{\lambda_{k m}(b)-\lambda}\right) v_{k m} e_{k m}, \\
\left\|\widetilde{K_{b}} u-\widetilde{K_{b+\Delta b}} u\right\|^{2} & =\frac{|\Delta b|^{2}}{|b(b+\Delta b)|^{2}} \sum_{0 \leq k \leq k_{0}} \frac{\left|v_{k m}\right|^{2}}{\left|\lambda_{k m}(b+\Delta b)-\lambda\right|^{2}} \frac{4 m^{2} \pi^{2}}{\left|\lambda_{k m}(b)-\lambda\right|^{2}} .
\end{aligned}
$$

If $b+\Delta b \in A_{\sigma}\left(\frac{1}{r}\right), 0 \leq k \leq k_{0}$, and $0<\sigma<1$, then

$$
\frac{\left|v_{k m}\right|^{2}}{\left|\lambda_{k m}(b+\Delta b)-\lambda\right|^{2}} \leq\left|v_{k m}\right|^{2} r^{2}(k+1)^{2+2 \sigma} \leq r^{2}\left(k_{0}+1\right)^{4}\left|v_{k m}\right|^{2} .
$$

The relation $\lim _{m \rightarrow \infty} \frac{4 m^{2} \pi^{2}}{\left|\lambda_{k m}(b)-\lambda\right|^{2}}=b^{2}$ and the condition $0 \leq k \leq k_{0}$ imply that the quantity

$$
\frac{4 m^{2} \pi^{2}}{\left|\lambda_{k m}(b)-\lambda\right|^{2} \mid}=\frac{4 m^{2} \pi^{2}}{\left|\frac{2 m \pi}{b}+a k(k+n-1)-\lambda\right|^{2}}
$$

is dominated by a constant $C\left(k_{0}\right)$ depending on $k_{0}$. Therefore,

$$
\begin{aligned}
& \frac{|\Delta b|^{2}}{|b(b+\Delta b)|^{2}} \sum_{0<k \leq k_{0}} \frac{\left|v_{k m}\right|^{2}}{\left|\lambda_{k m}(b+\Delta b)-\lambda\right|^{2}} \frac{4 m^{2} \pi^{2}}{\left|\lambda_{k m}(b)-\lambda\right|^{2}} \\
& \leq \frac{|\Delta b|^{2}}{|b(b+\Delta b)|^{2}} \sum_{0 \leq k \leq k_{0}} r^{2}\left(k_{0}+1\right)^{4} C\left(k_{0}\right)\left|v_{k m}\right|^{2} \\
& \quad \leq \frac{|\Delta b|^{2}}{|b(b+\Delta b)|^{2}} r^{2}\left(k_{0}+1\right)^{4} C\left(k_{0}\right) \sum_{0 \leq k \leq k_{0}}\left|v_{k m}\right|^{2} .
\end{aligned}
$$

Since

we arrive at the estimate

$$
\sum_{0 \leq k \leq k_{0}}\left|v_{k m}\right|^{2} \leq\|v\|^{2} \leq M^{2}\|u\|^{2}
$$

$$
\left\|\widetilde{K_{b+\Delta b}}-\widetilde{K_{b}}\right\|^{2} \leq \frac{|\Delta b|^{2}}{|b(b+\Delta b)|^{2}} M^{2} r^{2}\left(k_{0}+1\right)^{4} C\left(k_{0}\right) .
$$

We choose $\Delta b$ so as to satisfy the condition

$$
\frac{|\Delta b|^{2}}{|b(b+\Delta b)|^{2}} M^{2} r^{2}\left(k_{0}+1\right)^{4} C\left(k_{0}\right)<\varepsilon .
$$

Then $\left\|K_{b+\Delta b}-K_{b}\right\|<3 \varepsilon$. This shows that the operator-valued function $b \mapsto K_{b}$ is continuous on $A_{\sigma}\left(\frac{1}{r}\right)$. The lemma is proved.

Lemma 6. The spectrum $\sigma(K)$ of the compact operator $K$ depends continuously on $K$ in the space $\operatorname{Comp}(\mathcal{H})$ of compact operators on $\mathcal{H}$, in the sense that for any $\varepsilon>0$ there exists $\delta>0$ such that for all compact (and even bounded) operators $B$ with $\|B-K\|<\delta$ we have

$$
\sigma(B) \subset \sigma(K)+V_{\varepsilon}(0), \quad \sigma(K) \subset \sigma(B)+V_{\varepsilon}(0) .
$$

Here $V_{\varepsilon}(0)=\{\lambda \in \mathbb{C}|| \lambda \mid<\varepsilon\}$ is the $\varepsilon$-neighborhood of the point 0 in $\mathbb{C}$.

Proof. Let $K$ be a compact operator; we fix $\varepsilon>0$. The structure of the spectrum of a compact operator shows that there exists $\varepsilon_{1}<\varepsilon / 2$ such that $\varepsilon_{1} \neq|\lambda|$ for all $\lambda \in \sigma(K)$. Let $S=\left\{\lambda_{1}, \ldots, \lambda_{k}\right\}$ be the set of all spectrum points $\lambda$ with $|\lambda|>\varepsilon_{1}$, and let $V=\bigcup_{\lambda \in S \cup\{0\}} V_{\varepsilon_{1}}(\lambda)$. Then $V$ is a neighborhood of $\sigma(K)$, and $V \subset \sigma(K)+V_{\varepsilon}(0)$. By the well-known property of spectra (see, e.g., [2, Theorem 10.20]), there exists $\delta>0$ 
such that $\sigma(B) \subset V$ for any bounded operator $B$ with $\|B-K\|<\delta$. Moreover (see, e.g., [2, p. 293, Exercise 20]), the number $\delta>0$ can be chosen so that $\sigma(B) \cap V_{\varepsilon_{1}}(\lambda) \neq \varnothing$ for all $\lambda \in S \cup\{0\}$. Then for all bounded operators $B$ with $\|B-K\|<\delta$ the required inclusions $\sigma(K) \subset \sigma(B)+V_{2 \varepsilon_{1}}(0) \subset \sigma(B)+V_{\varepsilon}(0)$ and $\sigma(B) \subset V \subset \sigma(K)+V_{\varepsilon}(0)$ are fulfilled. The lemma is proved.

It is easy to deduce the following statement from Lemma 6 .

Proposition 1. The function $\rho(\lambda, K)=\operatorname{dist}(\lambda, \sigma(K))$ is continuous on $\mathbb{C} \times \operatorname{Comp}(\mathcal{H})$.

Proof. Suppose $\lambda \in \mathbb{C}, K \in \operatorname{Comp}(\mathcal{H})$, and $\varepsilon>0$. By Lemma 6 there exists $\delta>0$ such that for any operator $H$ lying in the $\delta$-neighborhood of $K,\|H-K\|<\delta$, the inclusions (15) are fulfilled; these inclusions directly imply the estimate $|\rho(\lambda, K)-\rho(\lambda, H)|<\varepsilon$. Then for all $\mu \in \mathbb{C}$ with $|\mu-\lambda|<\varepsilon$ and all $H$ with $\|H-K\|<\delta$ we have

$$
|\rho(\mu, K)-\rho(\lambda, H)| \leq|\rho(\mu, K)-\rho(\lambda, K)|+|\rho(\lambda, K)-\rho(\lambda, H)|<|\mu-\lambda|+\varepsilon<2 \varepsilon .
$$

Since $\varepsilon>0$ is arbitrary, the function $\rho(\lambda, K)$ is continuous. The proposition is proved.

Combining Proposition 5 and Lemma 5, we obtain the following fact.

Corollary 1. The function $\rho(\lambda, b)=\operatorname{dist}\left(\lambda, \sigma\left(K_{b}\right)\right)$ is continuous on $(\lambda, b) \in \mathbb{C} \times A_{\sigma}\left(\frac{1}{r}\right)$.

Now we are ready to prove Theorem 4.

Proof of Theorem 4. By Corollary 1, the function $\rho(1 / \nu, b)$ is continuous with respect to the variables $(\nu, b) \in(\mathbb{C} \backslash\{0\}) \times A_{\sigma}\left(\frac{1}{r}\right)$. Consequently, the set

$$
B_{r}=\left\{(\nu, b) \mid \rho(1 / \nu, b) \neq 0, \quad b \in A_{\sigma}\left(\frac{1}{r}\right)\right\}
$$

is measurable, and so is the set $B=\bigcup_{r} B_{r}$. Clearly, $B \subset E$ and $E=B \cup B_{0}$, where $B_{0}=E \backslash B$. Obviously, $B_{0}$ lies in the set $\mathbb{C} \times\left(\mathbb{R}^{+} \backslash A_{\sigma}\right)$ of zero measure (recall that, by Theorem $1 A_{\sigma}$ has full measure in $\mathbb{R}^{+}$). Since the Lebesgue measure is complete, $B_{0}$ is measurable. Thus, the set $E$ is measurable, being the union of two measurable sets. Next, by Theorem 3, for $b \in A_{\sigma}$ the section $E^{b}=\{\nu \in \mathbb{C} \mid(\nu, b) \in E\}$ has full measure, because its complement $\left\{1 / \nu \mid \nu \in \sigma\left(K_{b}\right)\right\}$ is at most countable. Therefore, the set $E$ is of full plane Lebesgue measure. The theorem is proved.

The following important statement is a consequence of Theorem 4.

Corollary 2. For a.e. $\nu \in \mathbb{C}$, problem (11), (2) has a unique periodic solution with almost every period $b \in \mathbb{R}^{+}$.

Proof. Since the set $E$ is measurable and has full measure, for a.e. $\nu \in \mathbb{C}$ the section $E_{\nu}=\left\{b \in \mathbb{R}^{+} \mid(\nu, b) \in E\right\}=\left\{b \in \mathbb{R}^{+} \mid 1 / \nu \notin \sigma\left(K_{b}\right)\right\}$ has full measure, and for such $b$ 's, problem (12), (2) has a unique periodic solution with period $b$. The corollary is proved.

I am sincerely grateful to E. Yu. Panov for his attention to my work.

\section{REFERENCES}

[1] I. P. Kornfel'd, Ya. G. Sinaŭ, and S. V. Fomin, Ergodic theory, "Nauka", Moscow, 1980; English transl., Grundlehren Math. Wiss., vol. 245, Springer-Verlag, New York, 1982. MR610981(83a:28017) MR0832433 (87f:28019)

[2] W. Rudin, Functional analysis, 2nd ed., McGraw-Hill, Inc., New York, 1991. MR 1157815|(92k:46001)

[3] M. A. Shubin, Pseudodifferential operators and spectral theory, "Nauka", Moscow, 1978; English transl., Springer-Verlag, Berlin, 1987. MR509034 (80h:47057), MR0883081 (88c:47105) 
[4] Dang Khanh Hoi, Periodic solutions for some nonlinear evolution systems of natural differential equations, Differential Equations and Related Problems (Moscow, 2004): Thesis, p. 48 (Russian)

[5] - On periodic solutions for some nonlinear evolution natural differential equations on multidimensional torus, Vestnik Novgorod. Gos. Univ. Ser. Tekhn. Nauki No. 28 (2004), 77-79. (Russian)

Division of Mathematical Analysis, Novgorod State University, Bol'Shaya St.-PeterburgSKaya Ulitsa 41, 173003, Velikil Novgorod, Russia

E-mail address: dangkhanhhoi@yahoo.com

Received 1/DEC/2005

Translated by A. PLOTKIN 